\title{
An eclipsing post common-envelope system consisting of a pulsating hot subdwarf $B$ star and a brown dwarf companion
}

\author{
V. Schaffenroth ${ }^{1,2}$, B. N. Barlow ${ }^{3}$, H. Drechsel ${ }^{1}$, and B. H. Dunlap ${ }^{4}$ \\ 1 Dr. Remeis-Observatory \& ECAP, Astronomical Institute, Friedrich-Alexander Universität Erlangen-Nürnberg, Sternwartstr. 7, \\ 96049 Bamberg, Germany \\ e-mail: veronika.schaffenroth@sternwarte.uni-erlangen.de \\ 2 Institute for Astro- and Particle Physics, University of Innsbruck, Technikerstr. 25/8, 6020 Innsbruck, Austria \\ 3 Department of Physics, High Point University, 833 Montlieu Ave, High Point, NC 27268, USA \\ 4 Department of Physics and Astronomy, University of North Carolina, Chapel Hill, NC 27599-3255, USA
}

Received 20 January 2015 / Accepted 14 February 2015

\begin{abstract}
Hot subdwarf B stars (sdBs) are evolved, core helium-burning objects located on the extreme horizontal branch. Their formation history is still puzzling because the $\mathrm{sdB}$ progenitors must lose nearly all of their hydrogen envelope during the red-giant phase. About half of the known sdBs are in close binaries with periods from $1.2 \mathrm{~h}$ to a few days, which implies that they experienced a common-envelope phase. Eclipsing hot subdwarf binaries (also called HW Virginis systems) are rare but important objects for determining fundamental stellar parameters. Even more significant and uncommon are those binaries containing a pulsating sdB, since the mass can be determined independently by asteroseismology. Here we present a first analysis of the eclipsing hot subdwarf binary V2008-1753. The light curve shows a total eclipse, a prominent reflection effect, and low-amplitude pulsations with periods from 150 to $180 \mathrm{~s}$. An analysis of the light- and radial velocity curves indicates a mass ratio close to $q=0.146$, an radial velocity semi-amplitude of $K=54.6 \mathrm{~km} \mathrm{~s}^{-1}$, and an inclination of $i=86.8^{\circ}$. Combining these results with our spectroscopic determination of the surface gravity, $\log g=5.83$, the best-fitting model yields an sdB mass of $0.47 M_{\odot}$ and a companion mass of $69 M_{\text {Jup. }}$. Because the latter mass is below the hydrogen-burning limit, V2008-1753 represents the first HW Vir system that is known to consist of a pulsating $\mathrm{sdB}$ and a brown dwarf companion. Consequently, it holds strong potential for better constraining models of sdB binary evolution and asteroseismology.
\end{abstract}

Key words. subdwarfs - binaries: eclipsing - binaries: spectroscopic - brown dwarfs - stars: fundamental parameters stars: oscillations

\section{Introduction}

Hot subdwarf B (sdB) stars are core helium-burning stars with very thin hydrogen envelopes that are found on the extreme horizontal branch (EHB; see Heber 2009, for a review). While the future evolution of sdB stars is quite certain (they will become white dwarfs), their prior evolutionary paths remain to be fully resolved. Nonetheless, one thing seems to be assured: the relatively high fraction of sdBs in binaries (Maxted et al. 2002; Napiwotzki et al. 2004) implies that binary interactions play a crucial role in their formation. Han et al. (2002, 2003, and references therein) propose several binary-related mechanisms as formation channels for sdB stars:

- common envelope ejection leading to short-period binaries with periods from 0.1-10 days with either white dwarf or low-mass main sequence companions. From binary population synthesis (BPSs; Han et al. 2002), a mass distribution that peaks sharply around $0.47 M_{\odot}$ is expected. This mass is called the canonical mass;

- stable Roche lobe overflow resulting in sdB binaries with masses around $0.47 M_{\odot}$ and orbital periods of 10-100 d;

- double helium white dwarf mergers giving rise to single sdB stars with a wider distribution of masses.

Common envelope (CE) evolution is believed to be an important process for a large number and wide diversity of binary stars.
In particular, this formation process is vital to compact binary systems, since many of them once must have been orders of magnitude wider than their present-day separations. Although BPS models are, in general, able to produce compact binaries through common envelope evolution, this important phase in binary evolution is still poorly understood (see Ivanova et al. 2013, for a review). Many of the CE-related parameters in BPS codes remain unconstrained by observations (Clausen et al. 2012), including common-envelope ejection efficiency, minimum mass for core He ignition, and envelope binding energy. Consequently, the investigation of post-CE systems, especially those containing pulsators or exhibiting eclipses, can improve our understanding of this highly important stage of stellar evolution.

Some have proposed that a planet or brown dwarf companion might also be able to trigger the loss of envelope mass in the red-giant phase of the sdB progenitor (Soker 1998; Nelemans \& Tauris 1998; Han et al. 2012). When the host star evolves and becomes a red giant, close substellar companions will be engulfed in a common envelope. The outcome of this interaction is unclear, but there are three distinct possibilities: the substellar object can either survive, merge with the core of the red giant, or evaporate. The latest two outcomes might provide an explanation for some of the observed single sdBs. Discoveries of brown dwarfs in close, eclipsing sdB systems (Geier et al. 2011; Schaffenroth et al. 2014b) support the idea that substellar companions are sufficient for ejecting the envelopes of red giants. 
This scenario is also supported by the discovery of a brown $\operatorname{dwarf}\left(M_{\mathrm{BD}}=0.053 \pm 0.006 M_{\odot}\right)$ in a close orbit $(0.08 \mathrm{~d})$ around a low-mass white dwarf (Maxted et al. 2006). Consequently, substellar companions are able to influence late stellar evolution.

Eclipsing binaries are of special interest because they allow the determination of the masses and radii of both components, as well as the period and separation of the system. The mass, in particular, is vital to constraining sdB formation models. Eclipsing sdB binaries with low-mass main sequence or brown dwarf companions (HW Vir systems) are easily recognized by the shapes of their light curves, which are dominated by both eclipses and a strong reflection effect due to the large temperature difference between both components. The amplitude of the reflection effect depends on the separation distance, the temperature of the $\mathrm{sdB}$, and the albedo of the companion. In addition to shedding light on sdB evolution, studies of these relatively rare, postcommon envelope systems can be used to constrain current models of common-envelope evolution (e.g., Zorotovic et al. 2010, for eclipsing WD binaries).

Additional information concerning the structure and evolution of sdBs may be found by studying their pulsations. The $\mathrm{sdBV}_{\mathrm{r}}$ stars, discovered by Kilkenny et al. (1997) and independently theoretically predicted by Charpinet et al. (1996), are low-amplitude, multi-mode pulsators with typical periods ranging between 80-600 s. Their pulsation amplitudes are generally a few mmag. The short periods, being of the order of and shorter than the radial fundamental mode for these stars, suggest that the observed modes are low-order, low-degree p-modes (Charpinet et al. 2000). The known $\operatorname{sdBV}_{\mathrm{r}}$ stars occupy a region in the $T_{\text {eff }}-\log g$ plane with effective temperatures between $28000 \mathrm{~K}$ and $36000 \mathrm{~K}$ and surface gravities $(\log g$ ) between 5.2 and 6.2. Only $10 \%$ of all stars falling in this region show pulsations. Moreover, also sdBs with slow high radial-order g-mode oscillations with periods in the range of 2000-8000 s and temperatures of $22000-29000 \mathrm{~K}$ were found (Green et al. 2003). In the overlap region hybrid pulsators showing $\mathrm{p}$-mode, as well as g-mode pulsations, also exist (Schuh et al. 2006). Asteroseismology of these stars allows for an independent and accurate determination of the sdB mass.

The problem with typical HW Vir systems has been that they are single-lined spectroscopic binaries (see e.g. Schaffenroth et al. 2013, 2014b), and as such it is normally not possible to derive a unique mass ratio. HW Vir systems harboring a pulsating sdB primary, however, offer additional possibilities, since the stellar properties can be constrained by the light curve and asteroseismological analyses. Until recently, only two HW Vir systems with pulsating sdBs were known. The first such object - NY Vir - was found to be a pulsating sdB in an eclipsing binary by Kilkenny et al. (1998). It shows more than 20 pulsation modes with amplitudes of several mmag. An astroseismic analysis was able to determine the stellar parameters of this system independently from the light curve analysis (Van Grootel et al. 2013). Østensen et al. (2010) found another pulsating sdB + dM HW Vir binary (2M1938+4603). Unfortunately, the amplitudes of the pulsations, which were detected by Kepler, are so small that they cannot be observed by ground-based telescopes. This star is therefore not an ideal target for asteroseismological modeling.

Van Noord et al. (2013) report the discovery of a promising new HW Virginis system, V2008-1753 (CV = $16.8 \mathrm{mag})$, which was found during an automatic search for variable stars conducted with the $0.4 \mathrm{~m}$ Calvin College Robotic Telescope in Rehoboth, New Mexico. Their relatively noisy light curve showed eclipses and a strong reflection effect. Interestingly, this
sdB binary has an orbital period of only $1.58 \mathrm{~h}$, the shortest period ever found in an HW Virginis system. Here we present the first thorough analysis of this unique system, along with the discovery of low-amplitude pulsations in the sdB primary. Section 2 describes the observational data. The analysis is explained in Sects. 3 (spectroscopy) and 4 (photometry). Evidence for the brown dwarf nature of the companion is discussed in Sect. 5. Finally, we end with conclusions and suggest further opportunities that are offered by this one-of-a-kind system.

\section{Observations}

\subsection{Time-series spectroscopy}

We used the Goodman Spectrograph on the 4.1-m SOuthern Astrophysical Research (SOAR) telescope to obtain time-series spectroscopy of V2008-1753 over a full orbital cycle and determine the orbital velocity of its primary sdB component. A 1.35" longslit and a $930 \mathrm{~mm}^{-1}$ VPH grating from Syzygy Optics, LLC, were employed to cover the spectral range $3600-5250 \AA$ with an approximate resolution of $3.8 \AA$ ( $0.84 \AA$ per binned pixel). The position angle was set to 197.8 degrees $\mathrm{E}$ of $\mathrm{N}$ so that we could place a nearby comparison star on the slit, with the intention of characterizing and removing instrumental flexure effects. In order to maximize our duty cycle, we binned the spectral images by two in both the spatial and dispersion directions and read out only a $2071 \times 550$ (binned pixels) subsection of the chip. Each exposure had an integration time of $90 \mathrm{~s}$, with $8 \mathrm{~s}$ of overhead between successive images, yielding a duty cycle of approximately $92 \%$. Overall, we acquired 70 spectra of V2008-1753 between 23:49:20.87 UT (2013-08-31) and 01:42:07.17 UT (201309-01). The airmass decreased from 1.22 to 1.03 over this time period. Upon completion of the time series, we obtained several FeAr comparison lamp spectra and quartz-lamp spectra for wavelength calibration and flat-fielding.

Standard routines in IRAF, primarily ccdproc, were used to bias-subtract and flat-field the spectral images. Given the low thermal noise in the spectrograph system, we did not subtract any dark frames, as we wanted to avoid adding noise to the images. We used apall to optimally extract one-dimensional spectra and subtract a fit to the sky background for both the target and constant comparison star. Finally, we wavelength-calibrated each spectrum using the master FeAr comparison lamp spectrum taken at the end of the time-series run. The resulting individual spectra of V2008-1753 had a signal-to-noise ratio (S/N) around 15-20 pixel $^{-1}$, while the individual spectra of the comparison star (which looked to be $\mathrm{G}$ or K-type), had a S/N twice as high.

\subsection{Time-series photometry}

High-precision photometry was acquired with SOAR/Goodman through $i^{\prime}$ and $g^{\prime}$ filters on 15/16 August 2013. Although our primary goal was to model the binary light curve, our secondary goal was to look for stellar pulsations, and thus we used an instrumental setup that had both a high duty cycle and a Nyquist frequency above those of most known sdB pulsation modes. We binned the images $2 \times 2$ and read out only a small $410 \times 540$ (binned pixel) subsection of the chip that included both the target and 10 comparisons stars with the same approximate brightness level. We achieved an $87 \%$ duty cycle with $25 \mathrm{~s}$ exposures for the $g^{\prime}$ light curve and a $81.4 \%$ duty cycle with $14 \mathrm{~s}$ exposures for the $i^{\prime}$ light curve. In both cases, we observed the field for at least one full orbital cycle near an airmass of 1.1. We 
concluded each night with a set of bias frames and dome flats. Thermal count rates were too low to warrant the acquisition of dark frames.

All object images were bias-subtracted and flat-fielded using IRAF's ccdproc package. We extracted light curves with an IDL program we wrote that uses the function APER, which is based on DAOPHOT (Stetson 1987). We produced light curves over a wide range of aperture radii and selected the aperture that maximized the $\mathrm{S} / \mathrm{N}$ in the light curve. To mitigate the effects of atmospheric extinction and transparency variations, we divided the light curve of V2008-1753 by the average of those of the constant comparison stars, after verifying they were indeed nonvariable. Residual extinction effects are often removed in light curves of this duration by fitting and dividing the light curve by the best-fitting parabola. However, given the large-amplitude binary signals present, this process would distort the actual stellar signal. Instead, we fit straight lines through points of the same phase and, informed by these fits, removed the overall "tilt" in each light curve. While not perfect, this process helps to mollify the effects of residual extinction variations. We then divided each light curve by its mean value and subtracted a value of one from all points to put them in terms of fractional amplitude variations.

\section{Spectroscopic analysis}

\subsection{Radial velocity curve}

Radial velocity shifts were determined by measuring the positions of the hydrogen Balmer profiles $\mathrm{H} \beta$ through $\mathrm{H} 9$; although $\mathrm{H} 10, \mathrm{H} 11$, and several He I lines were also present, they were too noisy to provide reliable positions. We used the MPFIT routine in IDL (Markwardt 2009), which relies on the LevenbergMarquardt method, to fit simple inverse Gaussians to the line profiles and determine their centroids. The only available guide star near our field was significantly redder than the sdB target, and, consequently, its use led to a gradual shift in the slit alignment over the course of our observations (Goodman had no atmospheric dispersion corrector at the time); this drifting results in a color-dependent velocity shift. Additionally, instrumental flexure as the Nasmyth cage rotates also affected the stars' alignment on the slit, although only slightly. We removed both of these time-dependent wavelength solution effects by tracking the absorption-line features in the constant comparison star. Figure 1 presents the resulting radial velocity curve for V2008-1753, plotted twice for better visualization

We again used MPFIT to fit a sine wave to the data and determine the semi-amplitude of the velocity variation; the orbital period and phase were fixed during this process. We derive an orbital velocity of $K=54.6 \pm 2.4 \mathrm{~km} \mathrm{~s}^{-1}$ for the sdB primary. Eccentric orbits were also fitted to the radial velocity curve, but as we currently have no reason to prefer them over $e=0$, we continue the analysis under the assumption that the orbit is circular. Residuals from the best-fitting sine wave are shown in the bottom panel of Fig. 1 and are consistent with noise. The mean noise level in the Fourier transform of the residual velocity curve is $2 \mathrm{~km} \mathrm{~s}^{-1}$.

\subsection{Atmospheric parameters}

In preparation for determining the atmospheric parameters of the $\mathrm{sdB}$, we first de-shifted all individual spectra according to our orbital solution above and then co-added them to improve the overall S/N. We fit synthetic spectra, which were calculated using local thermodynamical equilibrium model atmospheres with solar metallicity and metal line blanketing (Heber et al. 2000), to

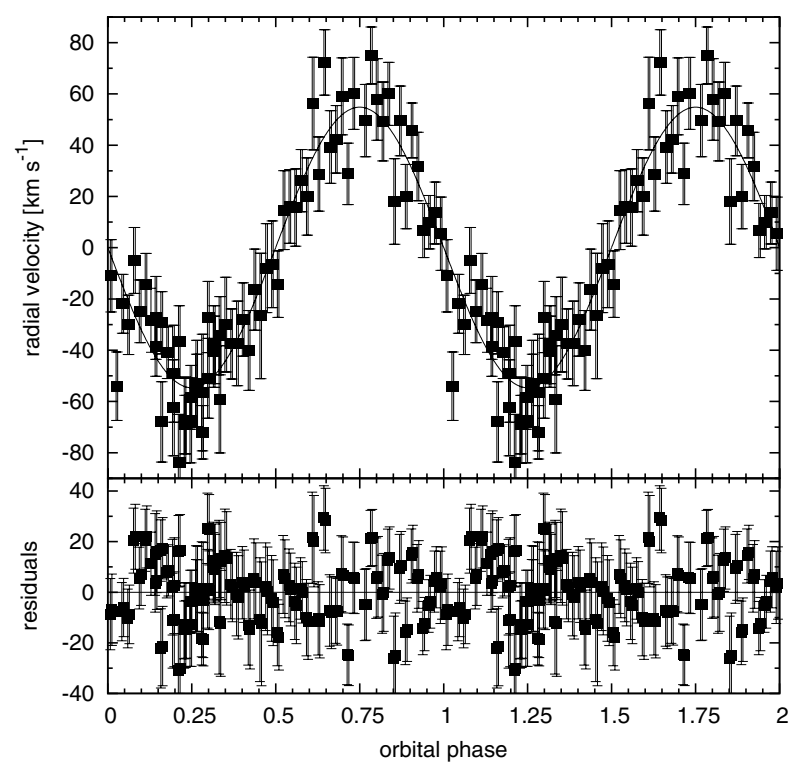

Fig. 1. Top panel: radial velocity curve for the $\mathrm{sdB}$ primary in V2008-1753, plotted twice for better visualization. The solid line denotes the best-fitting circular orbit to the data. Bottom panel: residuals after subtracting the best-fitting sine wave from the data.

the Balmer and helium lines of the co-added SOAR spectrum using SPAS (SPectral Analysis Software; Hirsch 2009). The bestfitting synthetic spectrum had the following orbital parameters:

$$
\begin{aligned}
T_{\text {eff }} & =32800 \pm 250 \\
\log g & =5.83 \pm 0.04 \\
\log y & =-2.27 \pm 0.13
\end{aligned}
$$

with $1 \sigma$ statistical errors determined by bootstrapping. For some sdB systems with reflection effects, an analysis of spectra with sufficiently high $\mathrm{S} / \mathrm{N}$ taken at different phases shows that the atmospheric parameters apparently vary with phase (e.g. Schaffenroth et al. 2013, 2014b). These variations can be explained by the companion's contribution to the spectrum (reflection only) varying with orbital phase. Systems with similar parameters show such variations in temperature and surface gravity on the order of $1000-1500 \mathrm{~K}$ and $0.1 \mathrm{dex}$, respectively. To account for the apparent change in the parameters we formally adopt the values determined from the co-added spectrum, which represents a mean value, with a larger error: $T_{\text {eff }}=32800 \pm 750 \mathrm{~K}$ and $\log g=5.83 \pm 0.05$ for the $\mathrm{sdB}$. Figure 2 shows the corresponding fit of the Balmer and helium lines. We excluded $\mathrm{H} \epsilon$ from the fit, as this line is mostly blended with the $\mathrm{Ca}$ II H-line and hence is less well represented by this fit.

The $T_{\text {eff }}-\log g$ diagram is displayed in Fig. 3 and shows that V2008-1753 lies in the middle of the extreme horizontal branch. Although it was previously suggested that HW Virginis systems cluster together only in a small part of the $T_{\text {eff }}-\log g$ diagram (Schaffenroth et al. 2014b), the position of V2008-1753 seems to go against this hypothesis. However, it is still apparent that most of the known HW Vir systems and reflection effect binaries (both $\mathrm{sdB}+\mathrm{dM}$ systems with different inclinations) concentrate in a distinct region between a $T_{\text {eff }}$ of $26000-30000 \mathrm{~K}$ and a $\log g$ of about 5.3 to 5.7 , only at the edge of the instability strip. There are five exceptions of binaries with sdOB star primaries and $\mathrm{M}$ star companions at higher temperatures, which are possibly just more evolved. Yet, the three HW Vir systems showing 


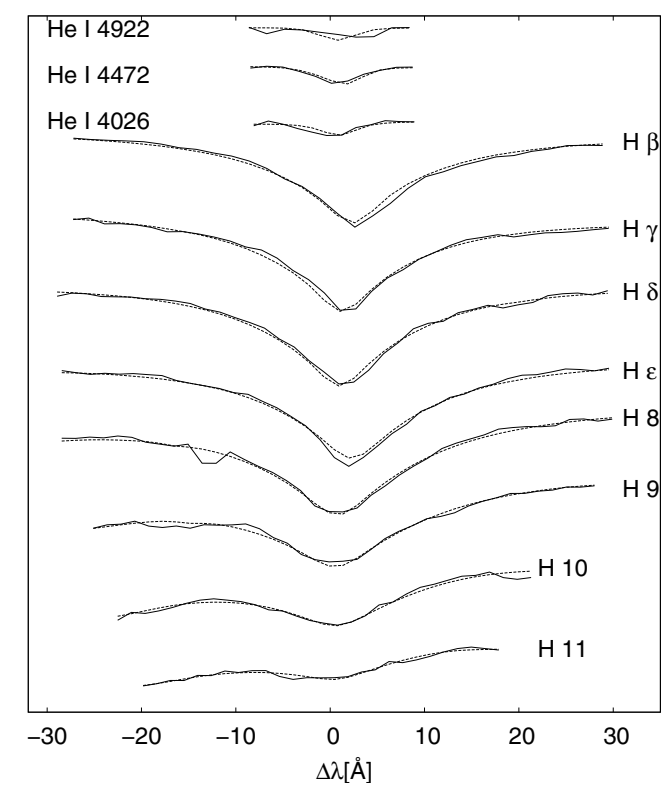

Fig. 2. Fit of the Balmer and helium lines in the co-added SOAR spectrum. The solid line shows the measurement, and the dashed line shows the best fitting synthetic spectrum. As the $\mathrm{H} \epsilon$ seems to be affected by a blend of the Ca line next to it, it was excluded from the fit.

short-period p-mode pulsations with amplitudes observable from the ground lie in a different part of the $T_{\text {eff }}-\log g$ diagram, nearer to the He-MS, in the central part of the instability strip for $\mathrm{sdBV}_{\mathrm{r}} \mathrm{s}$, as expected. In contrast to that the other sdB binaries with either white dwarf secondaries or companions of unknown type do not show any clustering but are uniformly distributed over the EHB. This could indicate that the sdBs with low-mass main sequence companions differ from the sdBs with white dwarf companions.

\section{Photometric analysis}

\subsection{Pulsations}

Both the $g^{\prime}$ and $i^{\prime}$ light curves from the SOAR telescope exhibit pulsations too low in amplitude to have been detected in the data analyzed by Van Noord et al. (2013). In order to disentangle the pulsations from the binary effects in the light curve, we used an approach similar to that demonstrated by Vučković et al. (2007). First, we fit the eclipses and reflection effect as described in Sect. 4.2 and subtracted the best-fitting model from the observed light curve. The original $g^{\prime}$ and $i^{\prime}$ filter light curves are displayed in Fig. 4, along with the same curves after the subtraction of the binary signal. The pulsations are visible by eye in the $g^{\prime}$ curve but less apparent in the $i^{\prime}$ data, due to its lower $\mathrm{S} / \mathrm{N}$ and lower pulsation amplitudes at redder wavelengths. The large-scale trends in both residual light curves are likely due to residual atmospheric extinction and transparency variations.

We calculated the Fourier transformations (FTs) of the light curves using FAMIAS ${ }^{1}$ (Zima 2008). The resulting FTs are displayed in Fig. 5. We detect at least four independent pulsation modes with periods ranging from 2.5 to $3 \mathrm{~min}$ and amplitudes $<4$ ppt. The best-fit frequencies, along with their amplitudes and $\mathrm{S} / \mathrm{N}$, are listed in Table 1 . The mode with the second-highest amplitude in the $g^{\prime}$ light curve (F2) was not clearly detected in the $i^{\prime}$ light curve, but its apparent absence

\footnotetext{
1 http://www. ster . kuleuven . be/ zima/famias
}

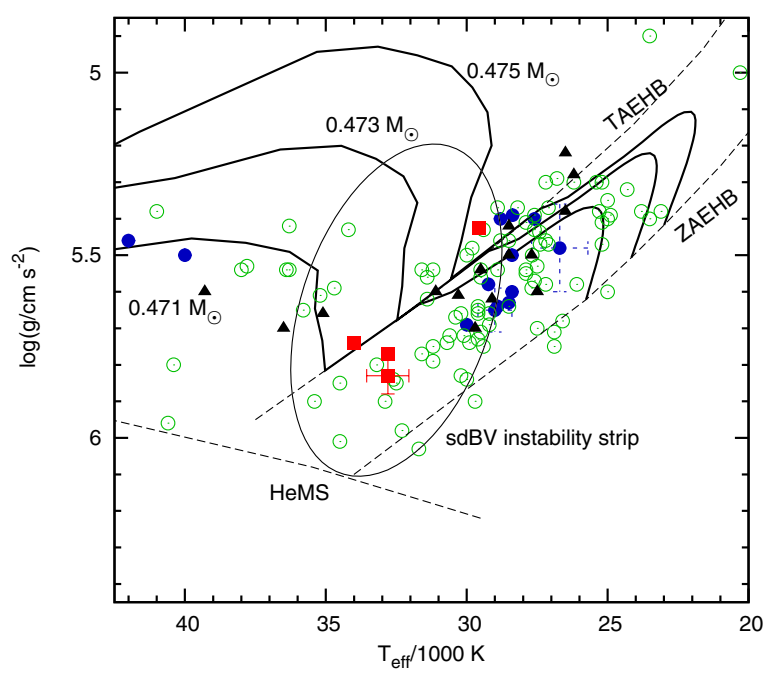

Fig. 3. $T_{\text {eff }}-\log g$ diagram of the HW Vir systems. The helium main sequence (HeMS) and the EHB band (limited by the zero-age EHB, ZAEHB, and the terminal-age EHB, TAEHB) are superimposed by evolutionary tracks by Dorman et al. (1993) for sdB masses of 0.471, 0.473 , and $0.475 M_{\odot}$. The positions of the HW Vir systems with pulsating sdBs - V2008-1753 (this work, with error bars), NY Vir (Van Grootel et al. 2013), 2M1938+4603 (Østensen et al. 2010), and PTF1 J072456+125301 (Schindewolf et al. 2015; Kupfer, priv. comm.) - are marked by red squares. Blue dots mark the positions of other HW Vir-like systems (Drechsel et al. 2001; For et al. 2010; Maxted et al. 2002; Klepp \& Rauch 2011; Østensen et al. 2008; Wood \& Saffer 1999; Almeida et al. 2012; Barlow et al. 2013; Schaffenroth et al. 2013, 2014a; Kupfer, priv. comm.). The positions of the two HW Vir systems with BD companions J1622 (Schaffenroth et al. 2014b) and J0820 (Geier et al. 2011) are indicated by the blue dots with error bars. The black triangles mark $\mathrm{sdB}+\mathrm{dM}$ systems showing a reflection effect but no eclipses (Kupfer et al. 2015, and references therein). The green, open dots represent other $\mathrm{sdB}+\mathrm{WD}$ binaries or $\mathrm{sdB}$ binaries with unknown companion type (Kupfer et al. 2015). The approximate location of the $\mathrm{sdBV}_{\mathrm{r}}$ instability strip is indicated by an ellipse.

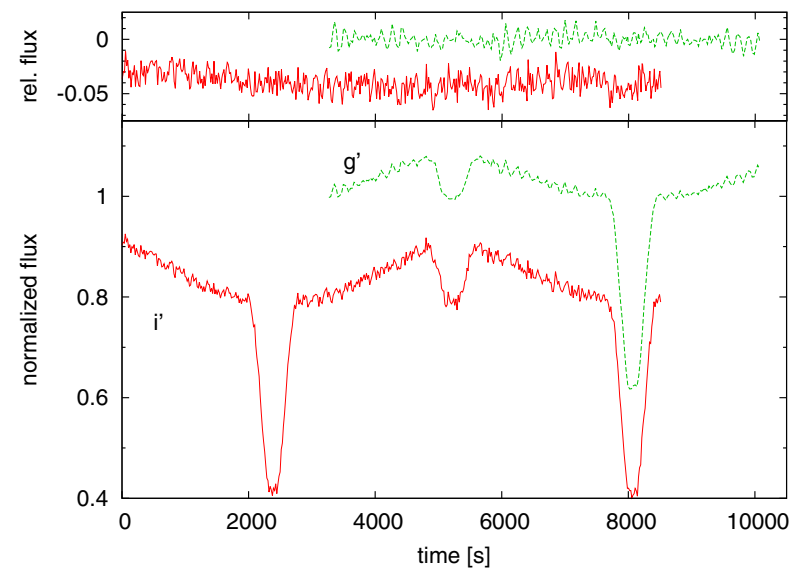

Fig. 4. $g^{\prime}$ (dashed line) and $i^{\prime}$ (solid line) filter light curves of V2008-1753 taken with SOAR. The two light curves were taken in subsequent orbital cycles. The sub-figure shows the pulsation signal after the subtraction of the binary signal by the best-fitting light curve model.

might be explained by the high noise level in this data set. The elevated power at lower frequencies is likely due to inaccuracies in the binary light curve modeling, along with the atmospheric extinction and transparency corrections. A much longer time base is needed to improve the frequency determination and to be able 

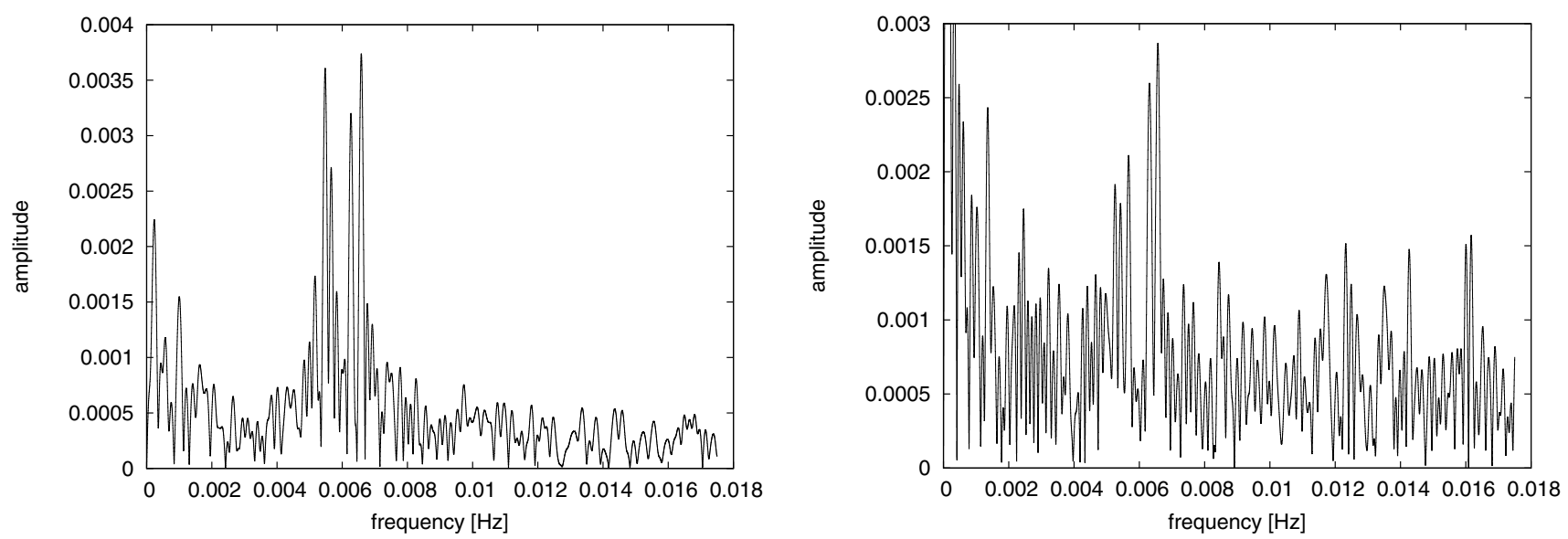

Fig. 5. Fourier transform of the pulsation signal shown in the sub-figure of Fig. 4. The left and right figures show the FTs of the $g^{\prime}$ and the $i^{\prime}$ filter light curves, respectively.

Table 1. Pulsation frequencies and amplitudes.

\begin{tabular}{|c|c|c|c|c|}
\hline & $\begin{array}{c}f^{a, b} \\
{[\mathrm{mHz}]}\end{array}$ & $\begin{array}{c}\text { Amplitude } \\
\text { [ppt] }\end{array}$ & Phase $^{c}$ & $S / N$ \\
\hline F1 & $6.565 \pm 0.005$ & $3.5 \pm 0.2$ & $0.739 \pm 0.019$ & 14.5 \\
\hline $\mathrm{F} 2$ & $5.494 \pm 0.008$ & $3.1 \pm 0.2$ & $0.82 \pm 0.03$ & 12.6 \\
\hline F3 & $6.289 \pm 0.006$ & $3.1 \pm 0.2$ & $0.74 \pm 0.02$ & 12.5 \\
\hline $\mathrm{F} 4$ & $5.638 \pm 0.011$ & $2.2 \pm 0.2$ & $0.71 \pm 0.05$ & 9.2 \\
\hline F1 & $6.572 \pm 0.006$ & $3.2 \pm 0.3$ & $0.12 \pm 0.03$ & 8.7 \\
\hline F2 & - & - & - & - \\
\hline F3 & $6.295 \pm 0.006$ & $2.8 \pm 0.3$ & $0.90 \pm 0.04$ & 7.6 \\
\hline F4 & $5.685 \pm 0.008$ & $2.3 \pm 0.3$ & $0.87 \pm 0.03$ & 6.2 \\
\hline
\end{tabular}

Notes. ${ }^{(a)}$ Upper half: frequencies found in $g^{\prime}$ light curve; lower half: frequencies found in $i^{\prime}$ light curve; ${ }^{(b)}$ errors as given by FAMIAS, more realistic is an error around $0.1-0.2 \mathrm{mHz}$; ${ }^{(c)}$ reference time: first point of light curve: $g^{\prime}: \mathrm{BJD}_{\mathrm{TBD}}=2456519.5846905 ; i^{\prime}: \mathrm{BJD}_{\mathrm{TBD}}=$ 2456520.53415896 .

to use the pulsations for asteroseismology. Consequently, we do not perform a more thorough pulsation analysis than this and limit our result simply to the detection of pulsations alone. To prepare the light curve for binary modeling, we use the results from Table 1 to subtract the detected pulsations from the original light curves.

\subsection{Binary light curve modeling}

The binary light curve exhibits all the typical features of an HW Vir system. Due to the short period and the relatively high temperature of the subdwarf, the reflection effect is rather strong, with an amplitude around 10\%. The secondary eclipse appears to be nearly total, in accordance with the high inclination and the very deep primary eclipse. As our light curves only cover one complete orbital cycle, it is not possible to determine an accurate period from our data alone. For the ephemeris we hence cite the period derived by Van Noord et al. (2013), which was determined from a much larger baseline. We were able to determine precise eclipse timings using our data by fitting parabolas to the minima. They are summarized in Table A.1. Using these values and the period from Van Noord et al. (2013), the ephemeris of the primary minimum is given by

$\mathrm{BJD}_{\mathrm{TBD}}=2456519.64027(1)+0.065817833(83) \cdot E$ where $E$ is the cycle number. The error in the period quoted by Van Noord et al. (2013) is likely to be an underestimate given the omission of systematics and the poor sampling. We believe an error of 0.0001 to be more appropriate. A comparison of the secondary eclipse compared to the primary eclipse of both light curves separately reveals a slight departure of the secondary mid-eclipse from phase 0.5 ; such an offset can be caused by both the Rømer delay (extra light travel time due to the binary orbit) and an eccentricity $e>0$. For small eccentricities, the total shift of the secondary eclipse with respect to phase 0.5 is given in Barlow et al. (2012):

$\Delta t_{\mathrm{SE}} \simeq \Delta t_{\mathrm{R} ø \mathrm{mer}}+\Delta t_{\mathrm{ecc}} \simeq \frac{P K_{\mathrm{sdB}}}{\pi c}\left(\frac{1}{q}-1\right)+\frac{2 P}{\pi} e \cos \omega$.

From both light curves we measure a shift of $3 \pm 1 \mathrm{~s}$ between the time of the secondary minimum and phase 0.5 . With our system parameters we would expect a theoretical shift of the secondary eclipse with respect to phase 0.5 due to the Rømer delay of $2 \mathrm{~s}$. If we take that into account, we would get a maximal eccentricity of $e \cdot \cos \omega<0.00055$. Ignoring the Rømer delay results in a maximum eccentricity of $e \cdot \cos \omega<0.0011$. Because of the large errors in the radial velocity determinations, the eccentricity cannot be constrained by the radial velocity curve to that precision.

A photometric solution to the binary light curve (with pulsations removed; Fig. 6) was determined using MORO (MOdified ROche program; see Drechsel et al. 1995). This program calculates synthetic light curves which we fit to the observations using the SIMPLEX algorithm. This light curve solution code is based on the Wilson-Devinney approach (Wilson \& Devinney 1971) but uses a modified Roche model that considers the mutual irradiation of hot components in close binary systems. More details of the analysis method are described in Schaffenroth et al. (2013). To calculate the synthetic light curves, $12+5 n$ ( $n$ is the number of light curves) parameters are used. Such a high number of partially-correlated parameters will inevitably cause severe problems if too many and wrong combinations are adjusted simultaneously. In particular, there is a strong degeneracy with respect to the mass ratio. After the orbital inclination, this parameter has the strongest effect on the light curve, and it is highly correlated with the component radii. Hence we kept the mass ratio fixed at certain values and calculated solutions for these mass ratios, which were subsequently compared and evaluated according to criteria explained below. 


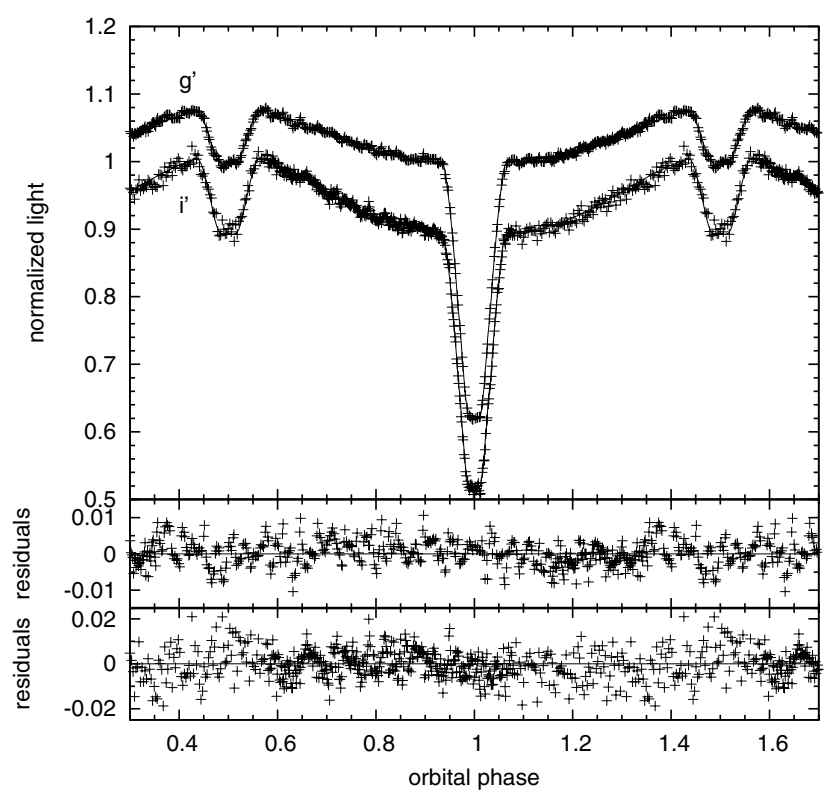

Fig. 6. $g^{\prime}$ and $i^{\prime}$ light curves after the removal of the pulsation signal as explained in Sect. 4.1 together with the best-fitting light curve model. The residuals displayed in the two lower panels still show signs of lowamplitude pulsations.

Given the large number of parameters present in the code, it is imperative to constrain as many parameters as possible based on independent inputs from spectroscopic analyses or theoretical constraints. From the spectroscopic analysis, we derived the effective temperature and the surface gravity of the sdB primary and fixed these parameters during the fitting. Due to the early spectral type of the primary star, the gravity darkening exponent was fixed at $g_{1}=1$, as expected for radiative outer envelopes (von Zeipel 1924). For the cool convective companion, $g_{2}$ was set to 0.32 (Lucy 1967). The linear limb darkening coefficients were extrapolated from the table of Claret \& Bloemen (2011).

To determine the quality of the light curve fit, the sum of squared residuals $\sigma$ of all observational points with respect to the synthetic curve was calculated as a measure of the goodness of the fit. Unfortunately, the $\sigma$ values of the best light curve fits for the different mass-ratios did not differ significantly. Therefore, we cannot determine a unique solution from the light curve analysis alone. The full set of parameters describing the best-fitting solution for a mass ratio of $q=0.146$, which corresponds to an sdB with the canonical mass of $0.47 M_{\odot}$ are given in Table A.2, with errors determined by the bootstrapping method. The light curves in the $g^{\prime}$ and $i^{\prime}$ bands are displayed in Fig. 6 together with the best-fit models for these parameters. The parameters of the system derived from this light curve solution together with the semi-amplitude of the radial velocity curve are summarized in Table 2. The errors result from error propagation of the errors in $K$ and $P$.

\section{The brown dwarf nature of the companion}

From the semi-amplitude of the radial velocity curve, the orbital period, and the inclination, we can derive the masses and radii of both components for each mass ratio. The masses follow from:

$M_{1}=\frac{P K_{1}^{3}}{2 \pi G} \frac{(q+1)^{2}}{(q \cdot \sin i)^{3}}$

$M_{2}=q \cdot M_{1}$
Table 2. Parameters of V2008-1753.

\begin{tabular}{c|c|c}
\hline \hline & \multicolumn{2}{c}{ V2008-1753 } \\
Coordinates & $200816.355-175310.52(\mathrm{~J} 2000.0)$ \\
\hline $\mathrm{cV}$ & {$[\mathrm{mag}]$} & 16.8 \\
$i$ & $\circ$ & $86.83 \pm 0.45$ \\
$K$ & {$\left[\mathrm{~km} \mathrm{~s}^{-1}\right]$} & $54.6 \pm 2.4$ \\
$P$ & {$[\mathrm{~h}]$} & $1.5796280 \pm 0.0000002$ \\
$M_{\text {sdB }}$ & {$\left[M_{\odot}\right]$} & $0.47 \pm 0.03$ \\
$M_{\text {comp }}$ & {$\left[M_{\odot}\right]$} & $0.069 \pm 0.005$ \\
$a$ & {$\left[R_{\odot}\right]$} & $0.56 \pm 0.02$ \\
$R_{\text {sdB }}$ & {$\left[R_{\odot}\right]$} & $0.138 \pm 0.006$ \\
$R_{\text {comp }}$ & {$\left[R_{\odot}\right]$} & $0.086 \pm 0.004$ \\
$\log g(\mathrm{sdB}$, phot $)$ & & $5.83 \pm 0.02$ \\
$\log g(\mathrm{sdB}$, spec $)$ & & $5.83 \pm 0.05$ \\
$T_{\text {eff,sdB }}$ & {$[\mathrm{K}]$} & $32800 \pm 750$ \\
\hline
\end{tabular}

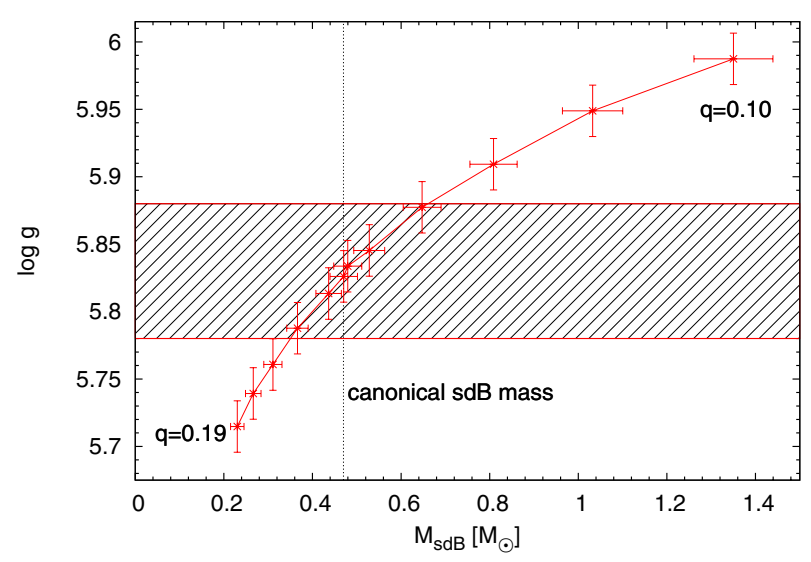

Fig. 7. Comparison of the photometric and spectroscopic surface gravity for the solutions with different mass ratio $q=0.10,0.11,0.12,0.13$, $0.14,0.145,0.146,0.15,0.16,0.17,0.18,0.19$ (marked by the error cross). The spectroscopic surface gravity with uncertainty is given by the shaded area.

and the fractional radii of the light curve solution together with

$a=\frac{P}{2 \pi} \frac{K_{1}}{\sin i} \cdot\left(\frac{1}{q}+1\right)$

yield the radii. For each mass ratio we get different masses and radii. It was stated already in Sect. 4.2 that it is not possible to determine the mass ratio from the light curve analysis alone. However, from the sdB mass and radius determined by the light curve analysis we can calculate a photometric surface gravity and compare that to the surface gravity derived by the spectroscopic analysis. The result is shown in Fig. 7. An agreement of spectroscopic and photometric surface gravity values is reached for solutions that result in $\mathrm{sdB}$ masses between 0.35 and $0.62 M_{\odot}$. It is therefore possible to find a self-consistent solution. This is a fortunate situation, because gravity derived from photometry was found to be inconsistent with the spectroscopic result in other cases, such as AA Dor (Vučković et al. 2008).

To constrain the solutions even more, we can also use theoretical mass-radius relations for the low-mass companions by Baraffe et al. (2003) and compare them to the masses and radii of the companion derived by the light curve solutions for the various mass ratios. This was done in a similar way as in Schaffenroth et al. (2014b). This comparison is displayed in Fig. 8. Relations for different ages of 1, 5 and $10 \mathrm{Gyr}$ were used. The measured mass-radius relation is well matched by 
theoretical predictions for stars $\gtrsim 3$ Gyr for companion masses between $0.056 M_{\odot}$ and $0.073 M_{\odot}$. The corresponding mass range for the sdB star extends from $0.35 M_{\odot}$ to $0.53 M_{\odot}$.

However, inflation effects have been found in the case of hot Jupiter exoplanets (e.g. Udalski et al. 2008) and also in the MS + BD binary CoRoT-15b (Bouchy et al. 2011). As the companion is exposed to intense radiation of a luminous hot star at a distance of only $0.56 R_{\odot}$, this effect cannot be neglected and would result in an underestimation of the radius, if compared to non-irradiated models (Baraffe et al. 2003). The maximum inflation effect can be estimated from the comparison of our solutions to the theoretical mass-radius relations shown in Fig. 8. This figure shows that an inflation of more than about $10 \%$ can be excluded, because otherwise none of the theoretical mass-radius relations would match the measured one, even if the star were as old as 10 Gyr.

The mass-radius relation for the companion would be in perfect agreement with the light curve solution for a companion with a mass of $0.069 M_{\odot}$, a radius of $0.086 R_{\odot}$, and an age of $\sim 5-10 \mathrm{Gyr}$, if we assume an inflation of $6-11 \%$. The corresponding mass of the $\mathrm{sdB}$ is $0.47 M_{\odot}$, exactly the canonical sdB mass, which we therefore adopt for the sdB throughout the rest of the paper. A similar result was found in the analysis of the sdB + BD binary J1622 (Schaffenroth et al. 2014b), which has a comparable period and parameters.

The companion has a mass below the limit for hydrogenburning and thus appear to be a brown dwarf - the third confirmed around a hot subdwarf star.

\section{Summary and conclusions}

We performed an analysis of the spectrum and light curve of V2008-1753 and find that this eclipsing binary consists of a pulsating sdB with a brown dwarf companion. This is the first system of this kind ever found. Similar to J1622, an inflation of the brown dwarf by more than about $10 \%$ can be excluded.

V2008-1753 has the shortest period of all known HW Vir systems and the second shortest period of any sdB binary discovered to date. Due to the small separation distance and high temperature of the $\mathrm{sdB}$, the amplitude of the reflection effect is relatively large (more than 10\%). Consequently, this system might provide the chance to detect and evaluate spectral features of the irradiated companion, similar to AA Doradus and HW Vir (Vučković et al. 2008, 2014). If the companion's spectral features are detected, the radial velocities of both components could be determined. We then could derive an unbiased mass ratio of the system and obtain a unique spectroscopic and photometric solution.

Higher quality and resolution spectra should also allow the detection of the Rossiter-McLaughlin (RM) effect (cp. Vučković et al. 2007). This effect is due to the selective blocking of the light of the rotating star during an eclipse. The amplitude is mainly depending on radius ratio, the rotational velocity of the primary star, and the inclination of the system. As our system has a high inclination and a high radius ratio, the expected amplitude is quite high. From the RM effect it is, hence, possible to determine the rotational velocity independent from spectral line modeling. With the help of the rotational velocity we can check, if the $\mathrm{sdB}$ is synchronized, which should be the case due to synchronization theories, but is currently under debate (Schaffenroth et al. 2014b).

The presence of stellar pulsations in the sdB offers further possibilities to characterize this system. Once the pulsation frequencies are fully resolved and their modes identified

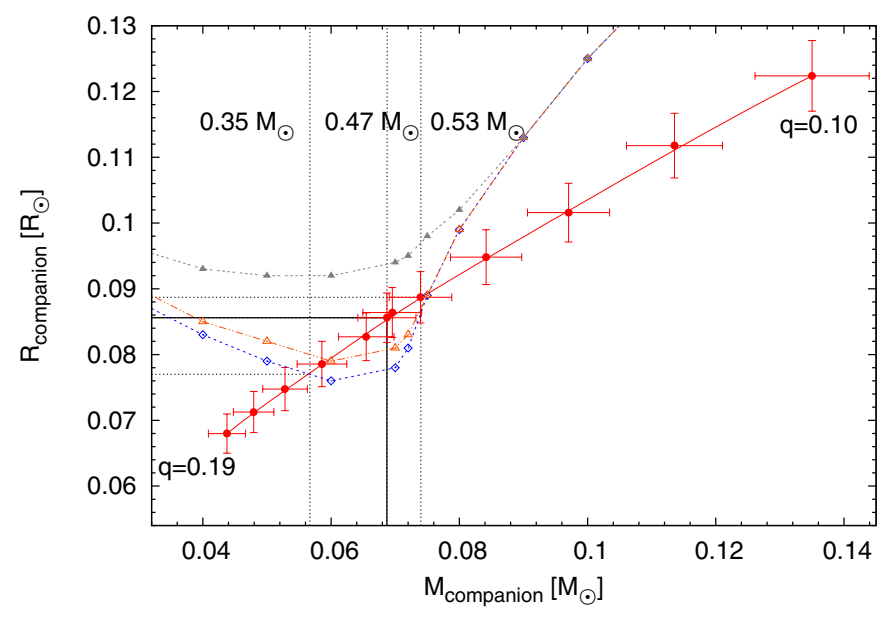

Fig. 8. Comparison of theoretical mass-radius relations of brown dwarfs by Baraffe et al. (2003) for an age of 1 Gyr (filled triangles), 5 Gyr (triangles) and $10 \mathrm{Gyr}$ (diamond) to results from the light cure analysis. Each error cross represents a solution from the light curve analysis for a different mass ratio $(q=0.10,0.11,0.12,0.13,0.14,0.145,0.146,0.15$, $0.16,0.17,0.18,0.19)$. The dashed vertical lines mark different values of the corresponding sdB masses. The solid line marks the most probable solution with $q=0.146$, which results in an sdB mass of $0.47 M_{\odot}$.

with higher-precision data over a longer time base, the mass and other properties of the sdB can be constrained by asteroseismology. These results can be compared to the light curve models. To date, only NY Vir (Van Grootel et al. 2013) has offered this opportunity. However, no signature of the companion could be identified in the spectrum of this system, and the masses from the light curve analysis remain biased.

The combined presence of pulsations and eclipses, furthermore, offers the possibility of eclipse mapping, as done for example for NY Vir (Reed et al. 2005; Reed \& Whole Earth Telescope Xcov 21 and 23 Collaborations 2006). Thereby, the eclipses can be used to determine pulsation modes, which are often difficult to uniquely identify. During the eclipse parts of the star are covered. Changing the amount and portion of regions of the star visible affects the pulsation amplitudes. The effect is changing for different pulsation modes, which can be identified in this way.

V2008-1753 has the potential to eventually replace NY Vir as the benchmark system for understanding sdB stars and their binary nature, if emission lines of the companion are detected. It would permit the direct comparison of independent techniques (namely light curve modeling, asteroseismology, spectroscopy, and radial velocity variations) used to derive the stellar parameters. Hence, the reliability of these methods and models could be checked as well as possible systematic errors of the derived parameters could be further investigated. Most notably, the mass determined by asteroseismology could be checked at a high precision level, if the semi-amplitudes of the radial velocity curves could be determined for both components.

Acknowledgements. Based on observations with the SOAR Telescope. The SOAR Telescope is a joint project of: Conselho Nacional de Pesquisas Cientificas e Tecnologicas CNPq-Brazil, The University of North Carolina at Chapel Hill, Michigan State University, and the National Optical Astronomy Observatory. Fourier transformation results obtained with the software package FAMIAS developed in the framework of the FP6 European Coordination Action HELAS (http://www.helas-eu.org/). V.S. acknowledges funding by the Deutsches Zentrum für Luft- und Raumfahrt (grant 50 OR 1110). 


\section{Appendix A: Results of the binary light curve modeling}

Table A.1. Eclipse times.

\begin{tabular}{ccc}
\hline \hline Filter & Eclipse & BJD $_{\mathrm{TBD}}[\mathrm{d}]$ \\
\hline$g^{\prime}$ & primary & $2456519.64027(1)$ \\
$g^{\prime}$ & secondary & $2456519.60733(2)$ \\
$i^{\prime}$ & primary & $2456520.56176(1)$ \\
$i^{\prime}$ & secondary & $2456520.59463(1)$ \\
$i^{\prime}$ & primary & $2456520.62757(1)$ \\
\hline
\end{tabular}

Table A.2. Adopted light curve solution.

\begin{tabular}{|c|c|c|}
\hline \multicolumn{3}{|c|}{ Fixed parameters } \\
\hline $\begin{array}{l}q\left(=M_{2} / M_{1}\right) \\
T_{\text {eff }}(1) \\
g_{1}{ }^{b} \\
g_{2}{ }^{b} \\
x_{1}\left(g^{\prime}\right)^{c} \\
x_{1}\left(i^{\prime}\right)^{c} \\
\delta_{2}{ }^{d}\end{array}$ & {$[\mathrm{~K}]$} & $\begin{array}{l}0.146 \\
33000 \\
1.0 \\
0.32 \\
0.21 \\
0.14 \\
0.0\end{array}$ \\
\hline \multicolumn{3}{|c|}{ Adjusted parameters } \\
\hline $\begin{array}{l}i \\
T_{\text {eff }}(2) \\
A_{1}{ }^{a} \\
A_{2}{ }^{a} \\
\Omega_{1}{ }^{f} \\
\Omega_{2}{ }^{f} \\
\frac{L_{1}}{L_{1}+L_{2}}\left(g^{\prime}\right)^{g} \\
\frac{L_{1}}{L_{1}+L_{2}}\left(i^{\prime}\right)^{g} \\
\delta_{1} \\
x_{2}\left(g^{\prime}\right) \\
x_{2}\left(i^{\prime}\right) \\
l_{3}\left(g^{\prime}\right)^{f} \\
l_{3}\left(i^{\prime}\right)^{f}\end{array}$ & $\begin{array}{c}\left.{ }^{\circ}\right] \\
{[\mathrm{K}]}\end{array}$ & $\begin{array}{l}86.83 \pm 0.45 \\
2960 \pm 550 \\
1.0 \pm 0.002 \\
1.2 \pm 0.05 \\
4.10 \pm 0.05 \\
2.389 \pm 0.008 \\
0.99995 \pm 0.00007 \\
0.99926 \pm 0.00068 \\
0.026 \pm 0.01 \\
0.44 \pm 0.06 \\
0.62 \pm 0.07 \\
0.007 \pm 0.001 \\
0.0 \pm 0.0\end{array}$ \\
\hline \multicolumn{3}{|l|}{ Roche radii $^{h}$} \\
\hline $\begin{array}{l}r_{1}(\text { pole }) \\
r_{1} \text { (point) } \\
r_{1}(\text { side }) \\
r_{1}(\text { back })\end{array}$ & $\begin{array}{l}{[\mathrm{a}]} \\
{[\mathrm{a}]} \\
{[\mathrm{a}]} \\
{[\mathrm{a}]}\end{array}$ & $\begin{array}{c}0.246 \pm 0.001 \\
0.249 \pm 0.002 \\
0.249 \pm 0.002 \\
0.249 \pm 0.002\end{array}$ \\
\hline $\begin{array}{l}r_{2}(\text { pole }) \\
r_{2} \text { (point) } \\
r_{2}(\text { side }) \\
r_{2}(\text { back })\end{array}$ & $\begin{array}{l}{[\mathrm{a}]} \\
{[\mathrm{a}]} \\
{[\mathrm{a}]} \\
{[\mathrm{a}]}\end{array}$ & $\begin{array}{l}0.150 \pm 0.001 \\
0.154 \pm 0.002 \\
0.152 \pm 0.001 \\
0.158 \pm 0.001\end{array}$ \\
\hline
\end{tabular}

Notes. ${ }^{(a)}$ Bolometric albedo. ${ }^{(b)}$ Gravitational darkening exponent. (c) Linear limb darkening coefficient; taken from Claret \& Bloemen (2011). ${ }^{(d)}$ Radiation pressure parameter, see Drechsel et al. (1995). ${ }^{(e)}$ Fraction of third light at maximum. ${ }^{(f)}$ Roche potentials. ${ }^{(g)}$ Relative luminosity; $L_{2}$ is not independently adjusted, but recomputed from $r_{2}$ and $T_{\text {eff }}(2)$. ${ }^{(h)}$ Fractional Roche radii in units of separation of mass centers.

\section{References}

Almeida, L. A., Jablonski, F., Tello, J., \& Rodrigues, C. V. 2012, MNRAS, 423, 478

Baraffe, I., Chabrier, G., Barman, T. S., Allard, F., \& Hauschildt, P. H. 2003, A\&A, 402, 701

Barlow, B. N., Wade, R. A., \& Liss, S. E. 2012, ApJ, 753, 101
Barlow, B. N., Kilkenny, D., Drechsel, H., et al. 2013, MNRAS, 430, 22

Bouchy, F., Deleuil, M., Guillot, T., et al. 2011, A\&A, 525, A68

Charpinet, S., Fontaine, G., Brassard, P., \& Dorman, B. 1996, ApJ, 471, L103

Charpinet, S., Fontaine, G., Brassard, P., \& Dorman, B. 2000, ApJS, 131, 223

Claret, A., \& Bloemen, S. 2011, A\&A, 529, A75

Clausen, D., Wade, R. A., Kopparapu, R. K., \& O’Shaughnessy, R. 2012, ApJ, 746, 186

Dorman, B., Rood, R. T., \& O’Connell, R. W. 1993, ApJ, 419, 596

Drechsel, H., Haas, S., Lorenz, R., \& Gayler, S. 1995, A\&A, 294, 723

Drechsel, H., Heber, U., Napiwotzki, R., et al. 2001, A\&A, 379, 893

For, B.-Q., Green, E. M., Fontaine, G., et al. 2010, ApJ, 708, 253

Geier, S., Schaffenroth, V., Drechsel, H., et al. 2011, ApJ, 731, L22

Green, E. M., Fontaine, G., Reed, M. D., et al. 2003, ApJ, 583, L31

Han, Z., Podsiadlowski, P., Maxted, P. F. L., Marsh, T. R., \& Ivanova, N. 2002, MNRAS, 336, 449

Han, Z., Podsiadlowski, P., Maxted, P. F. L., \& Marsh, T. R. 2003, MNRAS, 341, 669

Han, Z., Chen, X., Lei, Z., \& Podsiadlowski, P. 2012, in Fifth Meeting on Hot Subdwarf Stars and Related Objects, eds. D. Kilkenny, C. S. Jeffery, \& C. Koen, ASP Conf. Ser., 452, 3

Heber, U. 2009, ARA\&A, 47, 211

Heber, U., Reid, I. N., \& Werner, K. 2000, A\&A, 363, 198

Hirsch, H. 2009, Ph.D. Thesis, Friedrich Alexander Universität Erlangen Nürnberg

Ivanova, N., Justham, S., Chen, X., et al. 2013, A\&ARv, 21, 59

Kilkenny, D., Koen, C., O’Donoghue, D., \& Stobie, R. S. 1997, MNRAS, 285, 640

Kilkenny, D., O’Donoghue, D., Koen, C., Lynas-Gray, A. E., \& van Wyk, F. 1998, MNRAS, 296, 329

Klepp, S., \& Rauch, T. 2011, A\&A, 531, L7

Kupfer, T., Geier, S., Heber, U., et al. 2015, A\&A, 576, A44

Lucy, L. B. 1967, Z. Astrophys., 65, 89

Markwardt, C. B. 2009, in Astronomical Data Analysis Software and Systems XVIII, eds. D. A. Bohlender, D. Durand, \& P. Dowler, ASP Conf. Ser., 411, 251

Maxted, P. F. L., Marsh, T. R., Heber, U., et al. 2002, MNRAS, 333, 231

Maxted, P. F. L., Napiwotzki, R., Dobbie, P. D., \& Burleigh, M. R. 2006, Nature, 442,543

Napiwotzki, R., Karl, C. A., Lisker, T., et al. 2004, Ap\&SS, 291, 321

Nelemans, G., \& Tauris, T. M. 1998, A\&A, 335, L85

Østensen, R. H., Oreiro, R., Hu, H., Drechsel, H., \& Heber, U. 2008, in Hot Subdwarf Stars and Related Objects, eds. U. Heber, C. S. Jeffery, \& R. Napiwotzki, ASP Conf. Ser., 392, 221

Østensen, R. H., Green, E. M., Bloemen, S., et al. 2010, MNRAS, 408, L51

Reed, M. D., Brondel, B. J., \& Kawaler, S. D. 2005, ApJ, 634, 602

Reed, M. D., \& Whole Earth Telescope Xcov 21 and 23 Collaborations 2006, Mem. Soc. Astron. It., 77, 417

Schaffenroth, V., Geier, S., Drechsel, H., et al. 2013, A\&A, 553, A18

Schaffenroth, V., Geier, S., Barbu-Barna, I., et al. 2014a, in ASP Conf. Ser. 481, eds. V. van Grootel, E. Green, G. Fontaine, \& S. Charpinet, 253

Schaffenroth, V., Geier, S., Heber, U., et al. 2014b, A\&A, 564, A98

Schindewolf, M., Levitan, D., Heber, U., et al. 2015, A\&A, submitted

Schuh, S., Huber, J., Dreizler, S., et al. 2006, A\&A, 445, L31

Soker, N. 1998, AJ, 116, 1308

Stetson, P. B. 1987, PASP, 99, 191

Udalski, A., Pont, F., Naef, D., et al. 2008, A\&A, 482, 299

Van Grootel, V., Charpinet, S., Brassard, P., Fontaine, G., \& Green, E. M. 2013, A\&A, 553, A97

Van Noord, D. M., Molnar, L. A., \& Steenwyk, S. D. 2013 [arXiv: 1310. 0536] von Zeipel, H. 1924, MNRAS, 84, 665

Vučković, M., Aerts, C., Östensen, R., et al. 2007, A\&A, 471, 605

Vučković, M., Østensen, R., Bloemen, S., Decoster, I., \& Aerts, C. 2008, in Hot Subdwarf Stars and Related Objects, eds. U. Heber, C. S. Jeffery, \& R. Napiwotzki, ASP Conf. Ser., 392, 199

Vučković, M., Bloemen, S., \& Østensen, R. 2014, in ASP Conf. Ser. 481, eds. V. van Grootel, E. Green, G. Fontaine, \& S. Charpinet, 259

Wilson, R. E., \& Devinney, E. J. 1971, ApJ, 166, 605

Wood, J. H., \& Saffer, R. 1999, MNRAS, 305, 820

Zima, W. 2008, Commun. Asteroseismol., 157, 387

Zorotovic, M., Schreiber, M. R., Gänsicke, B. T., \& Nebot Gómez-Morán, A. 2010, A\&A, 520, A86 